\title{
reviscafuences
}

ISSN: $1575-7072$ | 8.15SN: 2172.7775

Páginas: 178-189

Recibido: 2020-10-09

Revisado: $2020-11-13$

Aceptado: 2021-03-12

Preprint: 2021-05-10

Publicación Final: 2021-05-15

www.revistascientificas.us.es/index.php/fuentes/index

DOI: https://doi.org/10.12795/revistafuentes.2021.12905

\section{Validez de Constructo y Confiabilidad de un Instrumento para Evaluar la Integración del Enfoque Socioformativo en las Prácticas Docentes en Instituciones de Educación Superior en México}

\author{
Construct Validity and Reliability of an Instrument to Evaluate the Integration of the \\ Socioformative Approach in Teaching Practices in Higher Education Institutions in \\ Mexico
}

\author{
(iD) Verónica Aguilar-Esteva \\ Universidad del Istmo (México) \\ iD Luis Gibran Juárez-Hernández \\ Centro Universitario CIFE (México) \\ (iD) Adán Acosta-Banda \\ Centro Universitario CIFE (México)
}

\begin{abstract}
En este artículo de presenta una Rúbrica analítica con el objetivo de evaluar el avance del enfoque socioformativo en las prácticas docentes en Instituciones de Educación Superior en México (IES), para este fin se utilizó la metodología instrumental aplicando la validez de constructo mediante el Anlalisis Factorial Exploratorio (AFE)- También se analizó la confiabilidad mediante el Alfa de Cronbach. La rúbrica se aplicó a 150 docentes de IES. El análisis de confiabilidad mostró un valor de Alfa de Cronbach de 0.930, el cual se considera adecuado. La prueba Kaiser Meyer Olkin (KMO:0.907) y esfericidad de Bartlett $(X 2: 997.351 \mathrm{Gl}: 36 ; p<0.000)$ indicaron la pertinencia de los datos para ser analizados mediante el AFE, revelando que todos los ítems se encuentran dentro del modelo denominado trabajo socioformativo (factor mayor a $64 \%$ de la varianza). Se recomienda realizar nuevos estudios que aporten conocimiento respecto al grado de avance en la aplicación de los ejes esenciales de la socioformación hacia la sociedad del conocimiento como son: la resolución de problemas del contexto mediante el trabajo colaborativo; el fomento al emprendimiento generando impacto en el entorno; el desarrollo del pensamiento crítico, sistémico, analítico, creativo; y la aplicación del proceso metacognitivo para mejora continua; así como, el mejoramiento de las condiciones de vida mediante la apropiación de los valores.

\section{Abstract}

In this article, the construct validity and reliability of an analytical rubric were analyzed- The objective of this research is to evaluate the progress of the socioformative approach in evaluating teaching practices in Higher Education Institutions (IES) in Mexico. The instrumental methodology was used, applying construct validity through Exploratory Factor Analysis (EFA). Reliability was also analyzed through Cronbach's Alpha. The rubric was applied to 150 teachers from HEl in Mexico. The reliability analysis showed a Cronbach's Alpha value of 0.930 , which is considered adequate. The Kaiser Meyer Olkin test (KMO: 0.907) and Bartlett's sphericity (X2: 997.351Gl: 36; $p<0.000)$ indicated the relevance of the data to be analyzed by EFA. The EFA revealed that all of the items are represented within the model called socioformative work (more than $64 \%$ of the variance); It is recommended to carry out new studies that provide knowledge regarding the degree of progress in the application of the essential axes of socioformation towards the knowledge society such as: the resolution of context problems through collaborative work; the promotion of entrepreneurship generating an impact on the environment; the development of critical, systemic, analytical, creative thinking; and the application of the metacognitive process for continuous improvement; as well as the improvement of living conditions through the appropriation of values.
\end{abstract}


Palabras clave

educación superior, prácticas docentes, sociedad del conocimiento, socioformación, validez de constructo.

Keywords

construct validity, higher education, educational practices, knowledge society, socioformation.

\section{Introducción}

En las Instituciones de Educación Superior (IES) se concentra gran parte de la responsabilidad de tener una comunidad preparada para enfrentar los retos de una sociedad en constante cambio (Cárdenas, Farías y Méndez, 2017; Fritsch y Aamoucke, 2017; Badillo, Buendía y Krüken, 2015; Tobón, 2017a); en este sentido, es necesario realizar mayores contribuciones en el área de la investigación para obtener conocimiento que coadyuve en la mejora de los procesos educativos existentes (Stehr, 1994). La educación superior en México no es obligatoria, lo que implica que no existe una normatividad definida respecto a los modelos que cada IES debe seguir. Sin embargo, la importancia de que estas instituciones logren la calidad educativa esperada representa para México un pilar para el desarrollo económico, social y cultural (Azamar-Alonso, 2015). Algunos de los modelos que más destacan en el ejercicio de los procesos de enseñanza-aprendizaje en la educación superior responden a momentos históricos de México, definidos por la situación social y el tipo de gobierno en el poder en ese momento.

Algunos ejemplos de modelos educativos que se han desarrollado con el fin de responder a las demandas de cada época son: el modelo tradicional de Sócrates, Platón y Aristóteles, dicho modelo surge en la antigua Grecia de forma innovadora para esa época como la primera manifestación del pensamiento sistémico, crítico y analítico alrededor de los años 400 a. C.; el modelo de pedagogía activa o reformista impulsado a principios del siglo XX por John Dewey quien estaba a favor de generar un modelo distinto de educación promoviendo que el alumno fuera descubriendo el conocimiento a partir de la observación y la comprobación, en uso de su inteligencia y libertad; el modelo de pedagogía tecnicista o conductista en el que se considera solo la importancia de generar conductas a partir del castigo o el estímulo utilizando las tecnologías sin importar el ser, al mismo tiempo, se enfoca en lograr las competencias para la actividad laboral y económica, surge a finales del siglo XIX con Iván Pávlov uniéndose a este enfoque Burrhus Frederic Skinner en el XX; el modelo personalizado cuyo precursor es Pierre Faure, surge a mediados del siglo XX con la consigna de dar libertad al estudiante de responsabilizarse de su avance en el aprendizaje a partir del autoconocimiento, la integración en la comunidad y el apoyo del maestro como guía; el modelo constructivista atribuido de manera formal a los aportes de Jean Piaget también a mediados del siglo XX en el que se enfatiza la importancia del conocimiento alcanzado previamente, aún antes de la adquisición del lenguaje mediante acciones sensoriales y motrices, en este modelo educativo el estudiante utiliza las herramientas para lograr el aprendizaje sin ser forzado ya que enfatiza en la necesidad o deseo personal de aprender; y el de competencias laborales promovida por la Organización para la Cooperación y el Desarrollo Económicos (OCDE) a partir de los años ochenta con el fin de invitar a las instituciones de educación a modificar sus currículos tomando en cuenta las competencias que son clave para el sector económico y laboral (Bañuelos, Sierra y Guzmán, 2011; Echavarría y De los Reyes, 2017).

Las prácticas docentes implementados en las IES en la actualidad deben ir evolucionando para poder responder a las demandas sociales de un mundo cambiante (Irigoyen, Jiménez y Acuña, 2011), es necesario identificar si éstos pueden enfrentar los retos que se plantea una nueva sociedad recientemente denominada sociedad del conocimiento, que consiste en la transformación de la sociedad a partir del trabajo colaborativo y la difusión masiva de información que permita resolver los problemas más relevantes relacionados con la preservación de la vida, con el uso de principios y valores éticos, aprovechando el uso de las Tecnologías de Información y Comunicación (TIC). Los problemas más relevantes en el contexto actual son: el calentamiento global, la pobreza, el hambre, el terrorismo, la discriminación, entre otros problemas globales (Tobón, 2017a). Es imperante lograr nuevas formas de investigar y de desarrollar el talento humano mediante la implementación de modelos que incluyan en sus ejes rectores el enfoque socioformativo; siendo este enfoque una propuesta que surge de la experiencia en el ramo educativo extendiéndose a otras áreas como las empresas, las instituciones no lucrativas, las instituciones gubernamentales, etc., desde el contexto Latinoamericano (Martínez, Tobón y Romero, 2017; Tobón, 2017a).

En la presente investigación se tiene un interés particular en el enfoque socioformativo, puesto que su origen se fundamenta en el contexto Latinoaméricano además, declara de forma explícita su orientación a formar para la sociedad del conocimiento (Abreu-Hernández y De la Cruz-Flores, 2015; Krüguer, 2006; Lee y Yang, 2000; Mansell y Wehn ,1998; Mora, 2004; Marcelo, 2001; Reaich, Gemino y Sauer, 2012; Tobón, Martínez, Valdez y Quiriz, 2018). Asimismo, se ha construido de forma colaborativa, con las contribuciones de académicos, autoridades educativas y expertos. Se enfoca en el desarrollo social sostenible articulando el trabajo con 
proyectos formativos (Tobón, 2017b) buscando el logro de retos que lleven a mejorar la calidad de vida y el cuidado del medio ambiente (Hernández-Mosqueda, Tobón-Tobón y Vázquez-Antonio, 2014; Tobón, 2017a; Tobón, Martínez, Valdez, y Quiriz, 2018). Este enfoque ha demostrado ser relevante y cada vez se aplica más en diversos países, principalmente Latinoamericanos (Aguilar-Esteva, 2019; Ambrosio, 2018; González-Peña, 2018; Herrera-Meza y Tobón, 2017; Martínez, Tobón y Romero, 2017; Parra-Acosta, López-Loya y Pizarr, 2018; Parra-Acosta, Tobón y López-Loya, 2015; Vázquez, Hernández, Vázquez-Antonio, Juárez-Hernández y Guzmán, 2017); se basa en priorizar la resolución de problemas del contexto en el ámbito educativo como fundamento de los procesos formativos, dejando atrás el aprendizaje de contenidos (Tobón, 2017a). Su fin en el contexto educativo es que los estudiantes desarrollen su talento resolviendo problemas prioritarios del entorno social en que viven, basándose en los postulados del pensamiento complejo, por lo cual propone la utilización del pensamiento analítico, el pensamiento crítico, el pensamiento sistémico, el emprendedurismo, la resolución de problemas del contexto, entre otras cosas (Cardona, Vélez y Tobón, 2016; Hernández-Mosqueda, TobónTobón y Vázquez-Antonio, 2014; Hernández, Tobón y Vázquez 2015; Hernández-Mosqueda, Tobón y Guerrero 2016; Tobón, González, Nambo y Vázquez-Antonio, 2015).

Las IES vienen implementando nuevos modelos educativos para afrontar los retos emergentes (Luna, 2019), pero esto ha tenido más dificultades que éxitos. Generalmente, se modifican las políticas institucionales, los procesos de construcción del currículo y la evaluación, pero luego esto se implementa poco en las clases con los estudiantes. Algunas causas de esto son: 1) la baja aceptación de las nuevas propuestas por parte de los docentes, porque creen que tienen planteamientos políticos contrarios o porque representan un deterioro a las IES; 2) las resistencias de los estudiantes, que creen que esto se hace para perjudicarlos; 3 ) la falta de formación continua, que vaya más allá de conferencias o pequeños talleres, y que posibilite apoyar a los docentes en la mejora; y 4) la ausencia de un proceso de construcción participativa con la comunidad universitaria. Respecto a la socioformación, diversas IES han comenzado su implementación (CIFE, 2015; Esteban-Rivera, 2017 y UTI, 2018) pero no se cuenta con instrumentos validados para determinar qué ejes de este enfoque han permeado en las prácticas docentes actuales, sin discriminar otros enfoques o paradigmas. Respecto a los instrumentos para evaluar o diagnosticar las prácticas docentes en los modelos educativos, se encontraron pocos en la literatura; algunos estudios que se encontraron y que se acercan al tipo de investigación propuesta en este documento, es el estudio descriptivo realizado en la Universidad Complutense de Madrid en el que se utilizó el cuestionario "Características de la Docencia Universitaria de Calidad desde el punto de vista de los Alumnos" (CDUCA); en este caso la evaluación del modelo, se hace desde las perspectivas de los estudiantes evaluando al profesorado universitario en nueve dimensiones: identidad personal y planificación; mediación del aprendizaje; disposición favorable hacia los estudiantes; utilización de las nuevas tecnologías; orientación, autoevaluación y control propio del aprendizaje; aprendizaje experiencial; dominio académico en su área y pensamiento crítico; esta investigación aborda dentro de estas nueve dimensiones dos que pueden asociarse al enfoque socioformativo, que son el aprendizaje experiencial y el dominio académico en su área y pensamiento crítico (Juanas y Beltrán, 2014). Todos estos instrumentos son escalas que dificultan la autoevaluación, porque los niveles con los cuales se evalúan los indicadores son subjetivos. Son necesarios nuevos instrumentos en forma de rúbricas, como la Rúbrica de Prácticas Pedagógicas (Tobón, Martínez, Valdez, y Quiriz, 2018), sin embargo, esta se enfoca a la educación media.

Por su parte, Dorantes y Tobón (2017) proponen la utilización de una rúbrica socioformativa con el fin de evaluar dentro de los modelos educativos, el desempeño del alumno, utilizando la taxonomía socioformativa y los niveles de consecución de resultados. En su propuesta las rúbricas proveen de información que facilita la identificación de las necesidades en el aula, previendo las consecuencias del desempeño de forma sistemática y permanente; a diferencia de la rúbrica diseñada en esta investigación, ya que esta se enfoca a evaluar la aceptación y adopción de los modelos educativos por parte de los docentes y administrativos. En este sentido la rúbrica conserva su relevancia para dicha evaluación, desde otro punto de referencia, sin embargo, converge en la evaluación del avance en el enfoque socioformativo en el ámbito educativo.

Tobón et al., (2018) analizan la forma en la que los procesos de enseñanza aprendizaje se centran tradicionalmente, y estos son: el plan de actividades didácticas, las evaluaciones de los alumnos, la asesoría, el apoyo al alumnado y el fomento de un ambiente agradable de trabajo. Sin embargo, destaca la poca importancia que se le da a las acciones que orientan a la formación de los ciudadanos que puedan enfrentar la nueva sociedad del conocimiento para ayudar a innovar el entorno Latinoamericano en el que destacan problemas de contexto muy relevantes que atañen a la población. Con este enfoque se diseñó y validó una rúbrica socioformativa que evalúa la práctica del docente con el fin de conocer en qué grado fomentan las prácticas basadas en los ejes rectores de la socioformación. Esta investigación aporta elementos interesantes a los que esta investigación suma aciertos, pudiendo generar un trabajo colaborativo que amplíe la visión de los resultados obtenidos en ambas investigaciones.

El Ministerio de Educación Nacional República de Colombia (2005) reporta resultados respecto a la calidad educativa asociada a la exitosa implementación de los modelos educativos en las regiones rurales, sin 
embargo, estas investigaciones se realizaron en el nivel básico, medio básico y medio superior, dejando un gran vacío en el estudio del nivel superior.

Los ejemplos de evaluaciones antes mencionados se diferencian del instrumento propuesto principalmente porque se enfocan en evaluar algunos casos y en otros niveles educativos que no son el superior, por otra parte, evalúan el desempeño del alumnado respecto al avance en el conocimiento de contenidos de los alumnos, por ende existen pocos estudios que se enfocan en evaluar de forma específica los ejes esenciales del enfoque socioformativo, abriéndose un área de investigación relacionada con la evaluación del avance del modelo socioformativo, por lo que surge la necesidad de contar con herramientas para dicha evaluación y que éstas sean válidas en términos de contenido y constructo.

\section{Metodología}

\subsection{Tipo de estudio}

El tipo de estudio es de naturaleza instrumental, el cual consiste en el desarrollo y/o adaptación de instrumentos, así como el análisis de sus propiedades psicométricas (Montero y León, 2015); su objetivo es el de proponer el diseño de un instrumento o la adaptación de una herramienta existente a un contexto distinto (Argumedo, Nóblega, Bárrig y Otiniano 2016). En este sentido, el instrumento fue sometido a la validez y a la confiabilidad. La validez de constructo consiste en verificar la consistencia que existe entre el perfil de referencia y la prueba, para sustentar el grado en el que los datos obtenidos en esta representan el atributo que se está evaluando. (Leyva, 2011). Por su parte, la confiabilidad se refiere a la consistencia o estabilidad de una medida cuando el proceso de medición se repite (Prieto y Delgado, 2010); en otras palabras, Magnusson (1978) la explica como la exactitud con que un instrumento logra medir lo que se requiere medir.

\subsection{Instrumentos}

Se realizó la validez de constructo y confiabilidad de la Rúbrica analítica para diagnosticar el avance en la integración de los ejes esenciales de la socioformación en las prácticas docentes en IES (CIFE, 2018), la cual fue diseñada y validada respecto a su contenido en un estudio previo (Aguilar-Esteva, Tobón-Tobón y JuárezHernández, 2019), obteniendo en el análisis valores mayores al mínimo esperado (V de Aiken>.75) en todos los ítems. El propósito de este instrumento es el de conocer el grado en el que el enfoque socioformativo está presente en las prácticas docentes en el nivel educativo superior actualmente. Se compone de nueve indicadores y cada indicador es evaluado mediante cinco niveles de desempeño basados en la taxonomía socioformativa: nivel muy bajo (preformal), nivel bajo (receptivo), nivel medio (resolutivo), nivel medio alto (autónomo) y nivel alto (estratégico). A su vez, cada nivel contiene descriptores que ayudan a determinar cuándo se ha logrado dicho nivel, aspecto del cual carecen las escalas. En la Tabla 1 se presentan los indicadores y sus descriptores.

\section{Tabla 1}

Rúbrica analítica para diagnosticar el avance en la integración de los ejes esenciales de la socioformación en las prácticas docentes en IES

\begin{tabular}{|c|c|}
\hline $\begin{array}{l}\text { Indicador } \\
\text { 1. ¿En qué grado en las clases se busca } \\
\text { resolver problemas del contexto? }\end{array}$ & $\begin{array}{l}\text { Respuestas } \\
\text { Preformal (Nivel muy bajo): En las clases se trabajan temas todo } \\
\text { el tiempo. Receptivo (Nivel bajo): En las clases se trabajan temas } \\
\text { y a veces se plantean juegos, retos, dinámicas y algunas aplicaciones. } \\
\text { Resolutivo (Nivel básico): En las clases se abordan problemas del } \\
\text { entorno, con base en necesidades puntuales. Autónomo (Nivel alto): } \\
\text { En las clases se busca que los estudiantes aprendan a identificar, } \\
\text { interpretar, argumentar y resolver problemas del contexto. Estratégico } \\
\text { (Nivel superior): En las clases se busca que los estudiantes logren metas } \\
\text { de aprendizaje resolviendo problemas del contexto y que esto permita } \\
\text { mejorar las condiciones de vida. }\end{array}$ \\
\hline
\end{tabular}

Continued on next page 
Table 1 continued

2. ¿En qué grado se promueve el trabajo colaborativo para lograr la resolución de problemas en las clases?

3. ¿En qué grado se busca formar para el emprendimiento, es decir, para generar proyectos de impacto en los problemas del entorno?

4. ¿En qué grado se busca en las clases que los estudiantes desarrollen el análisis crítico?

5. ¿En qué grado se busca en las clases el desarrollo del pensamiento sistémico?

6. ¿En qué grado se busca en las clases el desarrollo del pensamiento analítico?
Preformal (Nivel muy bajo): Durante las clases no se aborda el trabajo colaborativo. Receptivo (Nivel bajo): En las clases se hace trabajo en grupo, pero sin capacitación. Unos contribuyen y otros no. No hay manejo de roles en los equipos. Resolutivo (Nivel básico): En las clases hay algunas acciones de trabajo colaborativo, en las cuales los estudiantes buscan una meta común con las contribuciones de todos. Autónomo (Nivel alto): En las clases se busca el logro de metas comunes mediante las contribuciones articuladas de todos los estudiantes. Estratégico (Nivel superior): En las clases se lleva a cabo el trabajo colaborativo mediante el apoyo entre los mismos estudiantes para lograr las metas de aprendizaje. Los conflictos se resuelven y los equipos permanecen unidos a pesar de las dificultades.

Preformal (Nivel muy bajo): Las clases son tradicionalistas y centradas en el aprendizaje de contenidos. No hay formación para aprender a emprender. Receptivo (Nivel bajo): Las clases son centradas en contenidos, pero a veces se apoya a los estudiantes para que aprendan a resolver problemas y alcanzar metas. Resolutivo (Nivel básico): En las clases se apoya a los estudiantes para que planifiquen y ejecuten proyectos que contribuyan a la resolución de problemas del entorno. Autónomo (Nivel alto): En las clases se busca que los estudiantes autoevalúen los proyectos e implementan mejoras continuas. Estratégico (Nivel superior): En las clases se apoya a los estudiantes para que aprendan a resolver las dificultades que se presentan en todo proyecto y afronten la incertidumbre con estrategias flexibles y pensamiento positivo.

Preformal (Nivel muy bajo): En las clases se trabaja la exposición y recepción de temas y contenidos. No se aborda la formación del análisis crítico en los estudiantes. Receptivo (Nivel bajo): En las clases se abordan algunos elementos del análisis crítico, como por ejemplo identificar necesidades y problemas en el entorno, o determinar problemas en las políticas públicas, el campo profesional y la sociedad. Resolutivo (Nivel básico): Durante las clases los estudiantes aprenden a contrastar ideas, enfoques y modelos, para determinar aspectos comunes y diferentes. Autónomo (Nivel alto): Durante las clases los estudiantes aprenden a criticar los enfoques, modelos, metodologías y formas de abordar los problemas en el entorno. Estratégico (Nivel superior): Durante las clases los estudiantes aprenden a cuestionar y criticar con ecuanimidad, considerando los aspectos positivos y los aspectos a mejorar.

Preformal (Nivel muy bajo): Durante las clases los estudiantes y los profesores desconocen la aplicación del enfoque sistémico. Receptivo (Nivel bajo): Durante las clases se abordan los temas de cada contenido como un área única y separada del entorno. Resolutivo (Nivel básico): Durante las clases se abordan los temas procurando relacionar algunos temas con el entorno. Autónomo (Nivel alto): Durante las clases se abordan los temas relacionando varios sistemas que interactúan entre sí. Estratégico (Nivel superior): Durante todas las clases se enfatiza la interacción de los temas como sistemas que interactúan con otros sistemas. Preformal (Nivel muy bajo): Durante las clases solo se busca que el aprendizaje sea de contenidos de forma memorizada. Receptivo (Nivel bajo): Durante las clases algunos compañeros por iniciativa propia explican a otros lo que entendieron. Resolutivo (Nivel básico): Durante las clases el profesor invita a los alumnos a aplicar el conocimiento a casos reales sin dar seguimiento al resultado. Autónomo (Nivel alto): Durante las clases el profesor elabora actividades a partir de las cuales se realicen procesos de análisis y síntesis. Estratégico (Nivel superior): En todas las clases se aplica el pensamiento analítico a partir de procesos establecidos y actividades previamente pensadas y preparadas para realizar diferentes tipos de razonamiento. 
Table 1 continued

7. ¿En qué grado se busca en las clases el desarrollo del pensamiento creativo?

8. ¿En qué grado se busca en las clases que los estudiantes sean metacognitivos, es decir, que mejoren de manera continua hasta lograr las metas?

9. ¿En qué grado se busca en las clases el mejoramiento de las condiciones de vida mediante la aplicación de los valores?
Preformal (Nivel muy bajo): Durante las clases se abordan los temas de forma repetitiva y apoyado en contenidos. Receptivo (Nivel bajo): Durante las clases algunos alumnos aportan ideas originales. Resolutivo (Nivel básico): Durante las clases el aporte de ideas originales a partir del razonamiento es evaluado de forma positiva. Autónomo (Nivel alto): Durante algunas clases se fomenta el que los alumnos aporten ideas originales para la solución de problemas. Estratégico (Nivel superior): Durante las clases se establecen procesos de pensamiento creativo bien definidos en los que se desarrollan ideas originales para resolver problemas del contexto.

Preformal (Nivel muy bajo): Durante las clases no se fomentan actividades de mejora. Receptivo (Nivel bajo): Durante las clases los alumnos pueden presentar procesos de mejora sin que exista un proceso estructurado con dicho fin. Resolutivo (Nivel básico): Existen mecanismos para evaluar los procesos de mejora apegados a los métodos tradicionales de evaluación. Autónomo (Nivel alto): En las clases se fomenta como objetivo principal los procesos de mejora continua para lograr las metas. Estratégico (Nivel superior): Durante las clases además de fomentar actividades y evaluación de mejora continua, se da seguimiento y reconocimiento a la mejora continua.

Preformal (Nivel muy bajo): Durante las clases no se toman en cuenta las condiciones de vida ni se aplican los valores ya que esto no se encuentra en el contenido temático. Receptivo (Nivel bajo): Durante las clases se aplican los valores tradicionales de la cultura en las actividades sin enfatizar en las condiciones de vida o en el resultado de aplicarlos. Resolutivo (Nivel básico): Durante las clases se considera que el cuidado del medio ambiente es importante. Autónomo (Nivel superior): Durante las clases se elaboran proyectos para mejorar el medio ambiente. Estratégico: Durante las clases se da seguimiento al proceso de mejora del medio ambiente y de calidad de vida a partir de los conocimientos adquiridos y la solución de problemas del contexto.

De forma adicional, se empleó el Cuestionario de Factores Sociodemográficos (CIFE, 2017), con el fin de caracterizar a los participantes aplicando las siguientes preguntas: género; edad; condiciones económicas; estado civil; zona en la que vive (rural, urbana o semiurbana); si tiene hijos; en qué tipo de institución educativa realizó sus estudios (pública, privada o ambas); número de años de experiencia profesional como docente; número de horas al mes dedicadas a la formación continua sobre didáctica y evaluación del aprendizaje en los últimos tres años; último grado académico alcanzado.

\subsection{Participantes}

La rúbrica fue respondida por una muestra no probabilística de 150 docentes que laboran en cinco distintas IES. Los datos sociodemográficos se pueden observar en la Tabla 2.

Tabla 2

Datos Sociodemográficos de los docentes participantes

\begin{tabular}{|l|l|l|}
$\mathbf{N}$ & hombres & $\mathbf{1 5 0}$ \\
Edadnero & mujeres & $55.3 \%$ \\
& Promedio & $44.7 \%$ \\
\multirow{2}{*}{ Estado civil } & Desviación estándar & 43.7 \\
& Casado & 7.8 \\
& Soltero & $15.0 \%$ \\
& Divorciado & $10.7 \%$ \\
Número de hijos que ha tenido & Viudo & $2.0 \%$ \\
& Ninguno & $21.3 \%$ \\
& Uno & $23.3 \%$ \\
\hline
\end{tabular}

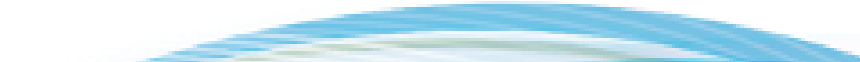


Table 2 continued

Número de años de experiencia profesional docente

Condiciones económicas

Zona de residencia

Tipo de institución en la realizó sus estudios

Promedio

Desviación estándar

Promedio

Desviación estándar

Excelentes

Buenas

Aceptables

Bajas

Zona urbana

Zona semiurbana

Zona rural

Institución pública

Institución privada

Ambas

Licenciatura completa

Maestría incompleta

Maestría completa

Doctorado incompleto

Doctorado completo

Posdoctorado completo
1.59

1.09

14.90

8.75

$0.7 \%$

$32.0 \%$

$62.0 \%$

$5.3 \%$

$82.0 \%$

$11.3 \%$

$6.7 \%$

$63.3 \%$

$11.3 \%$

$25.3 \%$

$12.0 \%$

$4.0 \%$

$51.3 \%$

$15.3 \%$

$16.0 \%$

$1.3 \%$

\subsection{Procedimiento}

Las etapas de la presente investigación fueron: 1) se aplicó la rúbrica a los participantes mediante un formulario en Google Formatos enviado por correo, previo consentimiento de querer participar en el estudio. Este instrumento se envió a 500 profesores de nivel educativo superior de los cuales solo 150 aceptaron participar en la investigación aportando sus respuestas; 2 ) se realizó el estudio de validez de constructo mediante el AFE, previo cumplimiento de los requisitos establecidos por esta técnica estadística; y 3) se analizó la confiabilidad mediante el Alfa de Cronbach (Cronbach, 1951). Los datos fueron procesados mediante el programa SPSS versión 19.

EI AFE es un procedimiento estadístico que destaca en la literatura para la contrastación de la validez de constructo (Pérez-Gil, Chacón y Moreno, 2000); es una técnica utilizada para examinar el cúmulo de variables y/o factores que explican las respuestas a los ítems de un instrumento. (Lloret-Segura, Ferreres-Traver, Hernández-Baeza, y Tomás-Marco, 2014). Además, se obtuvo la medida de adecuación de Kaiser-Meyer-Olkin que permite medir la adecuación del muestreo con el fin de evaluar si es conveniente aplicar el análisis factorial, considerándose apropiado si los valores resultantes fluctúan entre 0.5 y 1 (Montoya, 2007). Adicionalmente se aplicó la prueba de esfericidad de Bartlett que "se utiliza para probar la Hipótesis Nula que afirma que las variables no están correlacionadas en la población. Es decir, comprueba si la matriz de correlaciones es una matriz de identidad. Se puede dar como válidos aquellos resultados que nos presenten un valor elevado de la prueba y cuya fiabilidad sea menor a 0.05 " (Montoya, 2007). El AFE se llevó a cabo mediante la técnica de extracción de factores denominada extracción de factorización de eje principal dado que es un método robusto ante el supuesto de violación de normalidad, correlaciones moderadas y muestras pequeñas (Fabrigar, Wegener, MacCallum y Strahan, 1999; Gorsuch, 1983, De Winter y Dodou, 2012, Juárez-Hernández, 2018). Para la interpretación de los factores se estimó la matriz de factores con el fin de obtener las ponderaciones de cada variable respecto a su correlación con el factor seleccionado. Los valores altos indican una mayor contribución al factor analizado, mientras que los bajos indican baja representatividad (Méndez-Martínez y Rondón-Sepúlveda, 2012). Se calculó la varianza total explicada, se extrajo un factor dado que fue el único que obtuvo un autovalor mayor que uno (Méndez-Martínez y Rondón-Sepúlveda, 2012). La significación de las cargas factoriales pueden considerarse fuertes si éstas son mayores a 0.5 (Osborn y Castello, 2004). Finalmente, es importante tener en cuenta que los valores del Alfa de Cronbach fluctúan entre 0 y 1 , y mientras más se acerque el valor obtenido del análisis a 1 se podrá considerar que hay mayor consistencia interna dentro de la escala evaluada. George y Mallery (2003) recomiendan la siguiente escala para interpretar los valores resultantes: coeficiente alfa $>.9$ es excelente, coeficiente alfa $>0.8$ es bueno, coeficiente alfa $>0.7$ es aceptable, coeficiente alfa $>0.6$ es cuestionable, coeficiente alfa $>0.5$ es pobre, coeficiente alfa $<0.5$ es inaceptable.

\subsection{Aspectos Éticos}

La información recabada con el instrumento en las diferentes etapas del proceso metodológico de investigación fue obtenida con el consentimiento de los participantes quienes fueron informados del objetivo del instrumento 
y de la confidencialidad de la información obtenida. Se aclaró por escrito a los participantes que el instrumento en ningún momento serviría para afectar su desempeño como docente, ni tampoco en este caso se trataba de una prueba de inteligencia o personalidad. Para validar el consentimiento de los participantes se les solicitó que expresaran por escrito su conformidad en cuanto a que conocían el propósito del estudio y que aceptaban participar de forma voluntaria (Noreña, Alcaraz-Moreno, Rojas y Rebolledo-Malpica, 2012).

\section{Resultados}

Para el análisis de la validez de constructo de la rúbrica se realizó la revisión de la adecuación de los datos al análisis factorial. En primera instancia, se obtuvo la matriz de correlaciones (Tabla 3 en la que se observan valores positivos que superan 0.440 ; un determinante de la matriz de correlaciones con valor de 0.001 lo que nos indica que existen variables con intercorrelaciones muy altas, por lo que es factible continuar con el AF (Análisis Factorial).

Tabla 3

Matriz de correlaciones entre los ítems

\begin{tabular}{|l|l|l|l|l|l|l|l|l|l|}
\hline Ítem & $\mathbf{1}$ & $\mathbf{2}$ & $\mathbf{3}$ & $\mathbf{4}$ & $\mathbf{5}$ & $\mathbf{6}$ & $\mathbf{7}$ & $\mathbf{8}$ & $\mathbf{9}$ \\
1 & 1.000 & & & & & & & & \\
2 & $.723^{*}$ & 1.000 & & & & & & & \\
3 & $.701^{*}$ & $.766^{*}$ & 1.000 & & & & & & \\
4 & $.612^{*}$ & $.613^{*}$ & $.594^{*}$ & 1.000 & & & & & \\
5 & $.567^{*}$ & $.491^{*}$ & $.518^{*}$ & $.609^{*}$ & 1.000 & & & & \\
6 & $.463^{*}$ & $.456^{*}$ & $.484^{*}$ & $.679^{*}$ & $.721^{*}$ & 1.000 & & & \\
7 & $.447^{*}$ & $.572^{*}$ & $.572^{*}$ & $.620^{*}$ & $.627^{*}$ & $.660^{*}$ & 1.000 & & \\
8 & $.625^{*}$ & $.632^{*}$ & $.661^{*}$ & $.713^{*}$ & $.670^{*}$ & $.641^{*}$ & $.745^{*}$ & 1.000 & \\
9 & $.583^{*}$ & $.659^{*}$ & $.710^{*}$ & $.465^{*}$ & $.452^{*}$ & $.440^{*}$ & $.547^{*}$ & $.629^{*}$ & 1.000 \\
\hline
\end{tabular}

Nota: ${ }^{*} p<0.05$

La prueba Kaiser Meyer Olkin (KMO: .907) y esfericidad de Bartlett (X2: $997.351 \mathrm{Gl}: 36 ; p<0.000)$ indicaron la pertinencia de los datos para ser analizados mediante el AFE. El AFE en su primera matriz (comunalidades), reveló la representación de todos los ítems dentro del modelo factorial. Se indica que un factor explicó más del $64 \%$ de la varianza y este posee un autovalor superior a uno. De manera específica el análisis de la matriz factorial denotó la representación de los ítems con una carga significativa mayor a 0.5 (Osborn y Costello, 2004) a un solo factor (Tabla 4) según la varianza total explicada. En cuanto a la confiabilidad del instrumento se obtuvo un resultado óptimo, que fue de 0.930 medido por el Alfa de Cronbach.

Tabla 4

Comunalidades y cargas factoriales del instrumento

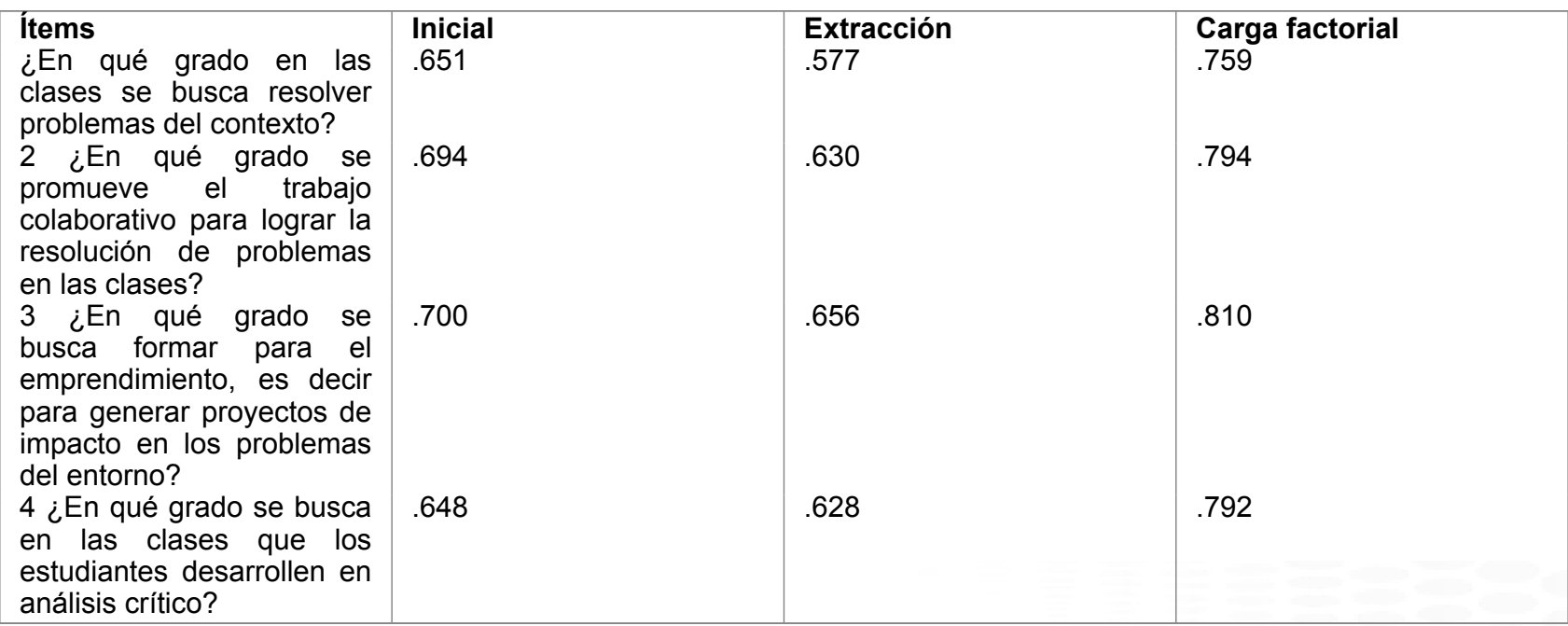




\section{Table 4 continued}

5 ¿En qué grado se busca en las clases el desarrollo del pensamiento sistémico?

6 ¿En qué grado se busca en las clases el desarrollo del pensamiento analítico? 7 ¿En qué grado se busca en las clases el desarrollo del pensamiento creativo? 8 ¿En qué grado se busca en las clases que los estudiantes sean metacognitivos, es decir, que mejores de manera continua hasta lograr las metas?

9 ¿En qué grado se busca en las clases el mejoramiento de las condiciones de vida mediante la aplicación de valores?
.625

.652

.728

.555

.745

.526

.725

.595

.771

.754

.868

.516

.718

\section{Discusión}

La Rúbrica analítica para diagnosticar el avance en la integración de los ejes esenciales de la socioformación en las prácticas docentes en IES fue primeramente validada en términos de redacción y de contenido con valores mayores al 0.80 en todos los ítems del instrumento respecto a la V. de Aiken (1985). A partir de los resultados óptimos obtenidos en esta fase se procedió a realizar la validez de constructo como se indica en este estudio, con este proceso se contribuye a la generación de instrumentos adecuados para conseguir información relevante en cuanto al avance de los ejes de la socioformación en los modelos educativos actuales. EI AFE fue realizado cumpliendo con el mínimo requerido de casos para la muestra que debe ser mayor a 50 (Lloret-Segura, Ferreres-Traver, Hernández-Baeza y Tomás-Marco, 2014) ya que el instrumento fue aplicado a 150 profesores, lo anterior proporciona certidumbre a los resultados obtenidos en el proceso de validación. Respecto al tipo de instrumento, las rúbricas son instrumentos que permiten entender claramente lo que se requiere responder, pues las opciones de respuestas que se enlistan contienen información suficiente para comprender cuál es la respuesta que más acertadamente responde a ítem en cuestión; por tal motivo, se puede realizar una valoración de las dimensiones adecuada, permitiendo una retroalimentación puntual y detallada de las evidencias recogidas (Hernández-Mosqueda et al., 2016). Los ítems planteados en la rúbrica van a permitir conocer el grado en el que desde la percepción de los profesores, los ejes esenciales el enfoque socioformativo son aplicados en los modelos actuales de las IES.

Dada la relevancia del enfoque y del tema de educación en México además de que la socioformación surge en un entorno educativo, tiene raíces latinoamericanas, que cada vez se posiciona más a partir del incremento de las investigaciones, aplicaciones, publicaciones, eventos académicos, entre otros (Tobón, 2017a), se diseñó, construyó y validó con buenos resultados un instrumento que permite evaluar el grado de avance en la integración en torno al enfoque socioformativo en las IES. Su identificación permitirá determinar los vacíos que existen respecto a dicho enfoque en las prácticas docentes en las IES actualmente y en consecuencia se tendrá una visión de los avances y de las estrategias que puedan implementarse para lograr mejores resultados.

\section{Conclusiones}

La importancia del presente estudio reside en que se ha obtenido evidencia de que la Rúbrica para diagnosticar el grado de avance en la integración de los ejes esenciales de la socioformación en las prácticas docentes en torno a los retos de la sociedad del conocimiento en el nivel educativo superior posee validez de constructo y confiabilidad, por tanto evalúa la presencia de los principales ejes de la socioformación en las prácticas docentes en nuevas investigaciones. 
Los resultados de la rúbrica fueron consistentes concluyendo que los ítems forman parte de un solo factor al que se le puede denominar trabajo socioformativo; esto con base en los contenidos de los ítems que indagan específicamente el avance de este enfoque en los modelos actuales; específicamente en temas como la resolución de problemas del contexto mediante el trabajo colaborativo; el fomento al emprendimiento generando impacto en el entorno; el desarrollo del pensamiento crítico, sistémico, analítico, creativo; y la aplicación del proceso metacognitivo para mejora continua; así como, el mejoramiento de las condiciones de vida mediante la aplicación de los valores.

Se propone seguir realizando trabajo de investigación para contribuir con nuevo conocimiento respecto al tema y para proponer estrategias que conlleven a la transformación de los modelos actuales de las IES y a la mejora continua, siendo relevante proseguir con una siguiente etapa que nos lleve al diagnóstico respecto al avance del enfoque socioformativo en estos modelos hacia la sociedad del conocimiento.

\section{Referencias}

Abreu-Hernández, L. F., y de la Cruz-Flores, G. (2015). Crisis en la calidad del posgrado ¿Evaluación de la obviedad, o evaluación de procesos para impulsar la innovación en la sociedad del conocimiento? Perfiles Educativos, 37(147), 162-182. Retrieved from https://dx.doi.org/10.1016/j.pe.2012.01.001 10.1016/j.pe.2012.01 .001

Aguilar-Esteva, V. (2019). Modelos Educativos en el Nivel Superior en México: Factores que favorecen y obstaculizan su Implementación. Atenas, 3(47), 18-33. Retrieved from https://bit.ly/2W5n5WH

Aguilar-Esteva, V., Tobón-Tobón, S., y Juárez-Hernández, L. G. (2019). Construcción y validación de instrumento para evaluar el avance del enfoque socioformativo y adopción de nuevos modelos educativos en el nivel superior en México. Revista ESPACIOS, 40(3).

Aiken, L. R. (1985). Three Coefficients for Analyzing the Reliability and Validity of Ratings. Educational and Psychological Measurement, 45(1), 131-142. Retrieved from https://dx.doi.org/10.1177/0013164485451012 $10.1177 / 0013164485451012$

Ambrosio-Prado, R. (2018). La socioformación: un enfoque de cambio educativo. Revista Iberoamericana de Educación, 76(1), 57-82. Retrieved from https://dx.doi.org/10.35362/rie7612955 10.35362/rie7612955

Ángel De-Juanas-Oliva, y Beltrán-Llera, J. A. (2013). Valoraciones de los estudiantes de ciencias de la educación sobre la calidad de la docencia universitaria. Educación XX1, 17(1), 59-82. Retrieved from https:// dx.doi.org/10.5944/educxx1.17.1.10705 10.5944/educxx1.17.1.10705

Argumedo, D., Nóblega, M., Bárrig, P., y Otiniano, F. (2016). Criterios Homologados de Investigación en Psicología (CHIP) Investigaciones instrumentales Versión 1.0, CHIP Instrumental. Comisión de investigación. Departamento de Psicología, 1-30. Retrieved from https://goo.gl/ktoJLM

Azamar-Alonso, A. (2015). El modelo educativo en México: una revisión de su alcance y una perspectiva para el futuro. Rostros, 17(31), 127-141. http://dx.doi.org/10.16925/ra.v17i31.1094

Badillo-Vega, R., Buendía, E., y Krücken, G. (2015). Liderazgo de los rectores frente a la "tercera misión de la universidad: Visiones globales, miradas locales. Revista Mexicana de Investigación Educativa, 20, 393-417.

Bañuelos, L. B., Sierra, J., y Guzmán, M. V. (2011). Recorrido histórico de los modelos educativos. In XV Congreso Internacional de Investigación en Ciencias Administrativas. La Administración y la Responsabilidad Social Empresarial (p. 444-460). Retrieved from https://goo.gl/eU1tnC

Cárdenas-Gutiérrez, C., Farías-Martínez, G. M., y Méndez-Castro, G. (2017). ¿Existe Relación entre la Gestión Administrativa y la Innovación Educativa? Un Estudio de Caso en Educación Superior / Is there a Relationship between Management and Educational Innovation? A Case Study at Higher Education Level. REICE. Revista Iberoamericana sobre Calidad, Eficacia y Cambio en Educación, 15.1(2017), 19-35. Retrieved from https://dx.doi.org/10.15366/reice2017.15.1.002 10.15366/reice2017.15.1.002

Cardona, S., Vélez, J., y Tobón, S. (2016). Contribution of socio-formative assessment to academic performance in an undergraduate program. Educar, 52(2), 423-423. 10.5565/rev/educar.763

CIFE. (2015). Modelo educativo centrado en la socioformación. Centro Universitario CIFE.

CIFE. (2017). Cuestionario de factores sociodemográficos. Centro Universitario CIFE.

CIFE. (2018). Ejes esenciales de la socioformación. México: Centro Universitario CIFE.

Cronbach, L. J. (1951). Coefficient alpha and the internal structure of tests. Psychometrika, 16(3), 297-334. Retrieved from https://dx.doi.org/10.1007/bf02310555 10.1007/bf02310555

de Educación Nacional República de Colombia., M. (Ed.). (2005). Evaluación de los modelos educativos que promueve el proyecto de educación rural del Ministerio de Educación Nacional. Retrieved from https://goo.gl/ 8r9Ep2

de Winter, J. C., y Dodou, D. (2012). Factor recovery by principal axis factoring and maximum likelihood factor analysis as a function of factor pattern and sample size. Journal of Applied Statistics, 39(4), 695-710. Retrieved from https://dx.doi.org/10.1080/02664763.2011.610445 10.1080/02664763.2011.610445

Dorantes-Nova, J. A., y Tobón-Tobón, S. . Y. (2017). Instrumentos de evaluación: Rúbricas socioformativas. 
Praxis Investigativa ReDIE, 9, 79-86.

Echavarría, L., y Reyes, C. D. L. (2017). El modelo de educación basada en competencias: genealogía, análisis y propuestas. XIV Congreso Nacional de Investigación Educativa-COMIE. San Luis Potosí, México, en. Retrieved from https://bit.ly/2WTWMQ7

Esteban-Rivera, E. R. (2017). Modelo Educativo, Universidad Nacional Hermilio Valdizán. Huánuco. Retrieved from https://bit.ly/3aLhFo4

Fabrigar, L. R., Wegener, D. T., MacCallum, R. C., y Strahan, E. J. (1999). Evaluating the use of exploratory factor analysis in psychological research. Psychological Methods, 4(3), 272-299. Retrieved from https:// dx.doi.org/10.1037/1082-989x.4.3.272 10.1037/1082-989x.4.3.272

Fritsch, M., y Aamoucke, R. (2017). Fields of knowledge in higher education institutions, and innovative startups: An empirical investigation. Papers in Regional Science, 96(1), 1-27.

George, D., y Mallery, P. (2003). SPSS for Windows step by step: A simple guide and reference. 11.0 update (4thed. Boston: Allyn \& Bacon.

González-Peña, L. (2018). UVE socioformativa: estrategia didáctica para evaluar la pertinencia de la solución a problemas de contexto. IE Revista de Investigación Educativa de la REDIECH, 9(16), 133-153. Retrieved from https://dx.doi.org/10.33010/ie_rie_rediech.v9i16.112 10.33010/ie_rie_rediech.v9i16.112

Gorsuch, R. (1983). Factor Analysis (Second Edition ed.). Lawrence Erlbaum Associates.

Hernández, J. S., Tobón, S., y Vázquez-Antonio, J. M. (2015). Estudio del liderazgo socioformativo mediante la cartografía conceptual. Revista Iberoamericana de Evaluación Educativa, 8(2), 105-128.

Hernández-Mosqueda, J. S., Tobón-Tobón, S., y Guerrero-Rosas, G. (2016). Hacia una evaluación integral del desempeño: las rúbricas socioformativas. Ra Ximhai, 12(6), 359-376. Retrieved from https://dx.doi.org/ 10.35197/rx.12.01.e3.2016.24.mh 10.35197/rx.12.01.e3.2016.24.mh

Hernández-Mosqueda, J. S., Tobón-Tobón, S., y Vázquez-Antonio, J. M. (2014). Estudio conceptual de la docencia socioformativa. Ra Ximhai, 10(5), 89-102. Retrieved from https://dx.doi.org/10.35197/rx.10.03.e1.2014 .06.jh 10.35197/rx.10.03.e1.2014.06.jh

Herrera-Meza, S. R., y Tobón, S. (2017). El director escolar desde el enfoque socioformativo. estudio documental mediante la cartografía conceptual. Revista de Pedagogía, 38, 164-194.

Horner-Reich, B., Gemino, A., y Sauer, C. (2012). Knowledge management and project-based knowledge in it projects: A model and preliminary empirical results. International Journal of Project Management, 30(6), 663-674. Retrieved from https://dx.doi.org/10.1016/j.ijproman.2011.12.003 10.1016/j.ijproman.2011.12.003

Irigoyen, J. J., Jiménez, M., y Acuña, K. F. (2011). Competencias y educación superior. Revista mexicana de investigación educativa, 16, 243-266.

Juárez-Hernández, L. G. (2018). Manual práctico de estadística básica para la investigación, $1^{2} E d$. Mount Dora, Florida: Kresearch.

Krüger, K. (2006). El concepto de sociedad del conocimiento. Revista Bibliográfica de Geografía y Ciencias Sociales, $X I, 683-683$.

Lee, C., y Yang, J. (2000). Knowledge Value Chain. Journal of Management Development, 19(9), 783-793.

Leyva-Barajas, Y. E. (2011). Una reseña sobre la validez de constructo de pruebas referidas a criterio. Perfiles Educativos, 33(131). Retrieved from https://bit.ly/2U18WaC

Lloret-Segura, S., Ferreres-Traver, A., Hernández-Baeza, A., y Tomás-Marco, I. (2014). El análisis factorial exploratorio de los ítems: una guía práctica, revisada y actualizada. Anales de Psicología, 30(3), 1151-1169. Retrieved from https://dx.doi.org/10.6018/analesps.30.3.199361 10.6018/analesps.30.3.199361

Luna, E. (2019). Evaluación formativa del modelo educativo en instituciones de educación superior en. México. RMIE, 24, 997-1026.

Magnusson, D. (1978). Teoría de los Test. Editorial Trillas. México.

Mansell, R., y Wehn, U. (1998). Knowledge Societies: Information Technology for Sustainable Development. New York: Oxford University Press.

Marcelo, C. (2001). Aprender a enseñar para la Sociedad del Conocimiento. Revista Complutense de Educación, 12(2), 531-593.

Martínez-Iñiguez, J., Tobón, S., y Romero-Sandoval, A. (2017). Problemáticas relacionadas con la acreditación de la calidad de la educación superior en América Latina. Innovación Educativa, 17, 79-96.

Méndez-Martínez, C., y Rondón-Sepúlveda, M. A. (2012). Introducción al análisis factorial exploratorio. Revista Colombiana de Psiquiatría, 41(1), 197-207. Retrieved from https://dx.doi.org/10.1016/s0034-7450(14)60077-9 $10.1016 / \mathrm{s} 0034-7450(14) 60077-9$

Modelo educativo, pedagógico y curricular. (2018). Ambato: UTI.

Montero, I., y León, G. (2005). Sistema de clasificación del método en los informes de investigación en psicología. International Journal of Clinical and Health Psychology, 5(1), 115-127.

Montoya, O. (2007). Aplicación del análisis factorial a la investigación de mercados. caso de estudio. Scientia et Technica, 23, 281-286.

Mora, J. G. (2004). La necesidad del cambio educativo para la sociedad del conocimiento. Revista 
Iberoamericana de Educación, 35(1), 13-37.

Noreña, A. L., Alcaraz-Moreno, N., Rojas, J. G., y Rebolledo-Malpica, D. (2012). Aplicabilidad de los criterios de rigor y éticos en la investigación cualitativa. AQUICHAN, 12(3), 263-274.

Osborne, J. W., y Costello, A. B. (2004). Sample size and subject to item ratio in principal components analysis. Research \& Evaluation, 9(11), 1-11.

Parra-Acosta, H., López-Loya, J., y Pizarro, N. (2018). Condiciones administrativas que propician el desempeño socioformativo de los médicos internos. IE Revista de Investigación Educativa de la REDIECH, 9(17), 119-132.

Retrieved from https://dx.doi.org/10.33010/ie_rie_rediech.v9i17.131 10.33010/ie_rie_rediech.v9i17.131

Parra-Acosta, H., Tobón, S., y López-Loya, J. (2015). Docencia socioformativa y desempeño académico en la educación superior. Paradigma, 36(1), 42-55. Retrieved from https://bit.ly/2ILmnGc

Pérez-Gil, J. A., Chacón-Moscoso, S., y Moreno-Rodríguez, R. (2000). Validez de constructo: el uso de análisis factorial exploratorio-confirmatorio para obtener evidencias de validez. Psicothema, 12(2), 442-446.

Prieto, G., y Delgado, A. (2010). Fiabilidad y validez. Papeles del Psicólogo, 31(1), 67-74.

Stehr, N. (1994). Knowledge societies. The transformation of labour, property and knowledge in contemporary society. London: Sage.

Tobón, S. (2017a). Essential axes of knowledge society and socioformation. Kresearch. Retrieved from https:// bit.ly/3cRsQxj

Tobón, S. (2017b). Evaluación socioformativa. Estrategias e instrumentos. Kresearch.

Tobón, S., González, L., Nambo, J., y Vázquez-Antonio, J. M. (2015). La Socioformación: Un Estudio Conceptual. Paradigma, 36(1), 7-29. Retrieved from en:shorturl.at/ilqTX

Tobón, S., Martínez, J. E., Valdez, E., y Quiriz, T. (2018). Prácticas pedagógicas: Análisis mediante la cartografía conceptual. Espacios, 39(45), 31. Retrieved from https://goo.gl/eLg48K

Vázquez-Antonio, J. M., Hernández-Mosqueda, J. S., Vázquez-Antonio, J., Juárez-Hernández, L. G., y Guzmán-Calderón, C. E. (2017). El trabajo colaborativo y la socioformación: un camino hacia el conocimiento complejo. Educación y Humanismo, 19(33), 334-356. Retrieved from https://dx.doi.org/10.17081/eduhum.19.33 .264810 .17081 /eduhum.19.33.2648 